\title{
Comparison of proton therapy and intensity modulated photon radiotherapy for locally advanced non-small cell lung cancer: considerations for optimal trial design
}

\author{
Taylor R. Cushman ${ }^{1}$, Vivek Verma ${ }^{2}$, Jean-Claude M. Rwigema ${ }^{3}$ \\ ${ }^{1}$ The University of Arizona College of Medicine-Phoenix, Phoenix, AZ, USA; ${ }^{2}$ Department of Radiation Oncology, University of Nebraska Medical \\ Center, Omaha, NE, USA; ${ }^{3}$ Department of Radiation Oncology, Mayo Clinic, Scottsdale, AZ, USA \\ Correspondence to: Jean-Claude M. Rwigema, MD. Department of Radiation Oncology, Mayo Clinic Arizona, 5777 E. Mayo Blvd, Phoenix, AZ 85054, \\ USA. Email: Rwigema.Jean@mayo.edu. \\ Provenance: This is an invited Editorial commissioned by the Section Editor Jun Zhou (Department of Nuclear Medicine, Zhongshan Hospital, \\ Fudan University, Shanghai, China). \\ Comment on: Liao Z, Lee JJ, Komaki R, et al. Bayesian Adaptive Randomization Trial of Passive Scattering Proton Therapy and Intensity-Modulated \\ Photon Radiotherapy for Locally Advanced Non-Small-Cell Lung Cancer. J Clin Oncol 2018. [Epub ahead of print].
}

Submitted Mar 13, 2018. Accepted for publication Mar 30, 2018.

doi: $10.21037 /$ jtd.2018.04.59

View this article at: http://dx.doi.org/10.21037/jtd.2018.04.59

The advent of proton therapy in the past years has generated many dosimetric studies comparing proton to photon radiation therapies (1-3). Proton therapy is able to deliver conformal, high dose radiation to the target site while sparing normal tissue. Although there is limited evidence on long term survival outcomes comparing photons versus protons (4-7), data suggests that proton therapy is better able to spare surrounding healthy tissue and thus has lower rates of adverse events (8-10). However, advances in technology have also led to the advent of intensity modulated radiation therapy (IMRT), which can deliver more conformal, highdose radiation compared to traditional three-dimensional conformal photon techniques $(11,12)$.

In lung cancer, pneumonitis, the most common radiation-related toxicity, negatively effects survival $(12,13)$. Previous research has demonstrated that there is a significant dose-dependent relationship between radiation dose to the lung and pneumonitis $(14,15)$.

As a result, IMRT and proton therapy have garnered interest as means of improving survival through both reduced toxicity rates and improved local control. To answer this important question, Liao et al. (16) initiated the first randomized controlled trial comparing IMRT and passive scattering proton therapy (PSPT) for locally advanced nonsmall cell lung cancer (NSCLC). The primary outcomes were radiation pneumonitis (RP) and local recurrence (LR).
The trial utilized Bayesian adaptive modeling to detect differences between treatments, if they existed, during the trial, in order to allocate more patients to the more beneficial treatment plan if a difference was observed. Patients with stage II to IIIB; or stage IV disease with either a single brain metastasis or recurrent disease after surgical resection eligible to receive definitive chemoradiotherapy, were recruited. From historical data, the study anticipated RP rates of $15 \%$ and $5 \%$ in the IMRT and PSPT groups, respectively $(11,17)$; LF rates were anticipated to be $25 \%$ in both groups. Each patient had evaluation of comparative PSPT and IMRT plans. If plans equally satisfied constraints on $\mathrm{V}_{20}$ and mean lung dose, patients were randomized into either treatment group. Both groups received either 66 or $74 \mathrm{~Gy}^{*}$ (relative biologic effectiveness, RBE).

Liao et al. (12) reported outcomes on 92 and 57 patients treated with IMRT and PSPT, respectively, with a median follow-up of 24.1 months. While there were no differences in mean doses to the lung or esophagus between groups, PSPT displayed reduced $V_{5-10}$ but increased lung $\mathrm{V}_{20-80}$. PSPT also had significantly lower mean heart dose $(\mathrm{P}=0.002)$. There were six patients in each group that experienced grade $\geq 3 \mathrm{RP}$ and no significant differences in RP between groups. One-year local failure rates were $10.9 \%$ for IMRT and 10.5 for PSPT $(\mathrm{P}=1.0)$. Median overall survival for IMRT and PSPT were 29.5 and 26.1 
months, respectively $(\mathrm{P}=0.297)$.

To evaluate the effect of time of enrollment in the trial, however, the researchers found significant differences in LF and RP when comparing results before versus after the midpoint of the study, despite similar clinical characteristics between respective groups. The early IMRT group had combined rates of LF and RP at 1 year of $21.1 \%$ vs. $18.2 \%$ in the latter group $(\mathrm{P}=0.047)$. Similarly, the early PSPT group had a combined rate of LF and RP at 1 year of $31 \%$ compared to $13.1 \%$ in the latter group $(\mathrm{P}=0.027)$. The authors attributed these findings to improved IMRT and PSPT plans as the trial went on; during the first year of trial initiation, an in-house automated optimization algorithm was added to the existing IMRT planning system, which improved plan quality (18). In post hoc analysis, new treatment plans were generated for six patients in the early PSPT group also showed improved quality, suggesting a learning curve may, in part, explain the differences in RP and LF by time of enrollment. Multivariable cox proportional hazards modeling revealed no other significant variables for RP or LF. Although, there were no improvements in dose-volume indices for lung, PSPT significantly reduced the mean radiation dose to the heart $(\mathrm{P}=0.002)$. Similarly, other studies have demonstrated lower doses to the heart, but suggested that lower mean lung doses and $V_{20}$ could also be achieved with improving planning and delivery techniques over time $(19,20)$.

In the era of combining radiation and immunotherapy, this is of particular interest. Research has demonstrated that proton therapy is associated with a $71 \%$ risk reduction in grade 4 lymphopenia (21), attributed to decreased irradiation of circulating lymphocytes. The increased conformity of proton therapy spares circulating lymphocytes through decreased radiation exposure and fractionation to major blood pools, such as the heart. Other research has shown that lymphopenia and post treatment circulating lymphocyte levels are independent predictors of survival $(22,23)$. These collective findings suggest that proton therapy may improve outcomes through lymphocyte sparing. Furthermore, given lymphocytes are the vehicle in which immunotherapy enhances anti-tumor effects, we hypothesize that proton therapy may better synergize with immunotherapy than photon therapy (24). In line with this hypothesis, a recent phase 2 trial comparing stereotactic proton versus photon therapy found that 3-year overall survival was $90 \%$ and $27.8 \%$, respectively (25). Unfortunately, the trial was closed early due to poor accrual, so the findings are incomplete.

Taken together, these findings suggest that IMRT and
PSPT may have similar toxicity profiles and rates of LR in locally advanced NSCLC, but optimization of plans remains paramount for optimal results. It is remarkable that differences in the plans over a short period of time, within the same institution, can result in drastic improvements in both LF and RP. Liao et al.'s (12) findings in the unplanned analysis bring to light a broader issue. With the rapidly increasing pace of technology development, clinicians are likely to be experience a learning curve upon implementation. Research investigating the efficacy of newly developed or implemented technologies must seriously consider and evaluate dosimetric changes in plans over time, among other variables.

\section{Acknowledgements}

None.

\section{Footnote}

Conflicts of Interest: The authors have no conflicts of interest to declare.

\section{References}

1. Kozak KR, Adams J, Krejcarek SJ, et al. A dosimetric comparison of proton and intensity-modulated photon radiotherapy for pediatric parameningeal rhabdomyosarcomas. Int J Radiat Oncol Biol Phys 2009;74:179-86.

2. Steneker M, Lomax A, Schneider U. Intensity modulated photon and proton therapy for the treatment of head and neck tumors. Radiother Oncol 2006;80:263-7.

3. Weber DC, Trofimov AV, Delaney TF, et al. A treatment planning comparison of intensity modulated photon and proton therapy for paraspinal sarcomas. Int J Radiat Oncol Biol Phys 2004;58:1596-606.

4. Eaton BR, Esiashvili N, Kim S, et al. Clinical Outcomes Among Children With Standard-Risk Medulloblastoma Treated With Proton and Photon Radiation Therapy: A Comparison of Disease Control and Overall Survival. Int J Radiat Oncol Biol Phys 2016;94:133-8.

5. Sato M, Gunther JR, Mahajan A, et al. Progression-free survival of children with localized ependymoma treated with intensity-modulated radiation therapy or protonbeam radiation therapy. Cancer 2017;123:2570-8.

6. Holliday EB, Frank SJ. Proton radiation therapy for head and neck cancer: a review of the clinical experience to date. Int J Radiat Oncol Biol Phys 2014;89:292-302. 
7. Bishop AJ, Greenfield B, Mahajan A, et al. Proton beam therapy versus conformal photon radiation therapy for childhood craniopharyngioma: multi-institutional analysis of outcomes, cyst dynamics, and toxicity. Int J Radiat Oncol Biol Phys 2014;90:354-61.

8. Timmermann B, Schuck A, Niggli F, et al. Spot-scanning proton therapy for malignant soft tissue tumors in childhood: First experiences at the Paul Scherrer Institute. Int J Radiat Oncol Biol Phys 2007;67:497-504.

9. Merchant TE, Hua CH, Shukla H, et al. Proton versus photon radiotherapy for common pediatric brain tumors: comparison of models of dose characteristics and their relationship to cognitive function. Pediatr Blood Cancer 2008;51:110-7.

10. Lomax AJ, Cella L, Weber D, et al. Potential role of intensity-modulated photons and protons in the treatment of the breast and regional nodes. Int J Radiat Oncol Biol Phys 2003;55:785-92.

11. Murshed H, Liu HH, Liao Z, et al. Dose and volume reduction for normal lung using intensity-modulated radiotherapy for advanced-stage non-small-cell lung cancer. Int J Radiat Oncol Biol Phys 2004;58:1258-67.

12. Liao ZX, Komaki RR, Thames HD Jr, et al. Influence of technologic advances on outcomes in patients with unresectable, locally advanced non-small-cell lung cancer receiving concomitant chemoradiotherapy. Int J Radiat Oncol Biol Phys 2010;76:775-81.

13. Bradley JD, Paulus R, Komaki R, et al. Standard-dose versus high-dose conformal radiotherapy with concurrent and consolidation carboplatin plus paclitaxel with or without cetuximab for patients with stage IIIA or IIIB non-smallcell lung cancer (RTOG 0617): a randomised, two-by-two factorial phase 3 study. Lancet Oncol 2015;16:187-99.

14. Marks LB, Bentzen SM, Deasy JO, et al. Radiation dosevolume effects in the lung. Int J Radiat Oncol Biol Phys 2010;76:S70-6.

15. Wang S, Liao Z, Wei X, et al. Analysis of clinical and dosimetric factors associated with treatment-related pneumonitis (TRP) in patients with non-small-cell lung cancer (NSCLC) treated with concurrent chemotherapy and three-dimensional conformal radiotherapy (3D-CRT).
Int J Radiat Oncol Biol Phys 2006;66:1399-407.

16. Liao Z, Lee JJ, Komaki R, et al. Bayesian Adaptive Randomization Trial of Passive Scattering Proton Therapy and Intensity-Modulated Photon Radiotherapy for Locally Advanced Non-Small-Cell Lung Cancer. J Clin Oncol 2018. [Epub ahead of print].

17. Sejpal S, Komaki R, Tsao A, et al. Early findings on toxicity of proton beam therapy with concurrent chemotherapy for nonsmall cell lung cancer. Cancer 2011;117:3004-13.

18. Zhang X, Li X, Quan EM, et al. A methodology for automatic intensity-modulated radiation treatment planning for lung cancer. Phys Med Biol 2011;56:3873-93.

19. Rwigema JM, Verma V, Lin L, et al. Prospective study of proton-beam radiation therapy for limited-stage small cell lung cancer. Cancer 2017;123:4244-51.

20. Berman AT, Teo BK, Dolney D, et al. An in-silico comparison of proton beam and IMRT for postoperative radiotherapy in completely resected stage IIIA non-small cell lung cancer. Radiat Oncol 2013;8:144.

21. Shiraishi Y, Fang P, Xu C, et al. Severe lymphopenia during neoadjuvant chemoradiation for esophageal cancer: A propensity matched analysis of the relative risk of proton versus photon-based radiation therapy. Radiother Oncol 2017. [Epub ahead of print].

22. Wild AT, Herman JM, Dholakia AS, et al. LymphocyteSparing Effect of Stereotactic Body Radiation Therapy in Patients With Unresectable Pancreatic Cancer. Int J Radiat Oncol Biol Phys 2016;94:571-9.

23. Wild AT, Ye X, Ellsworth SG, et al. The Association Between Chemoradiation-related Lymphopenia and Clinical Outcomes in Patients With Locally Advanced Pancreatic Adenocarcinoma. Am J Clin Oncol 2015;38:259-65.

24. Ebner DK, Tinganelli W, Helm A, et al. The Immunoregulatory Potential of Particle Radiation in Cancer Therapy. Front Immunol 2017;8:99.

25. Nantavithya C, Gomez DR, Wei X, et al. A Phase II Study of Stereotactic Body Radiotherapy and Stereotactic Body Proton Therapy for High-Risk Medically Inoperable Early-Stage Non-Small Cell Lung Cancer. Available online: http://www.redjournal.org/article/S0360$3016(18) 30316-\mathrm{X} / \mathrm{pdf}$
Cite this article as: Cushman TR, Verma V, Rwigema JM. Comparison of proton therapy and intensity modulated photon radiotherapy for locally advanced non-small cell lung cancer: considerations for optimal trial design. J Thorac Dis 2018;10(Suppl 9):S988-S990. doi: 10.21037/jtd.2018.04.59 